\title{
Morphological Transformation Network of Nanoparticles via DFT Simulations
}

\author{
J. A. S. Laranjeira ${ }^{a}$, G. S. L. Fabris ${ }^{\mathrm{a}, \mathrm{b}}$, M. M. Ferrer ${ }^{\mathrm{c}}$, A. R. Albuquerque ${ }^{\mathrm{d}}$, J. R. \\ Sambrano ${ }^{\mathrm{a}^{*}}$ \\ *email: jr.sambrano@unesp.br \\ $a$ - Modeling and Molecular Simulation Group, São Paulo State University, Bauru, SP, Brazil \\ b - Materials Science and Engineering Postgraduate Program, Department of Materials Engineering, \\ Federal University of Rio Grande do Norte, 59078-970 Natal, Brazil. \\ c - Graduate Program in Materials Science and Engineering, Center for Technology Development, \\ Federal University of Pelotas, 96010-610 Pelotas, RS, Brazil \\ d-Chemistry Institute, Federal University of Rio Grande do Norte, 59078-970 Natal, RN, Brazil
}

\section{SUPPORTING INFORMATION}


Figure $\mathrm{S} 1$ shows the lattice parameters and band gap energy $\left(E_{g a p}\right)$ deviation obtained from different functionals concerning the experimental results (reference [43]).

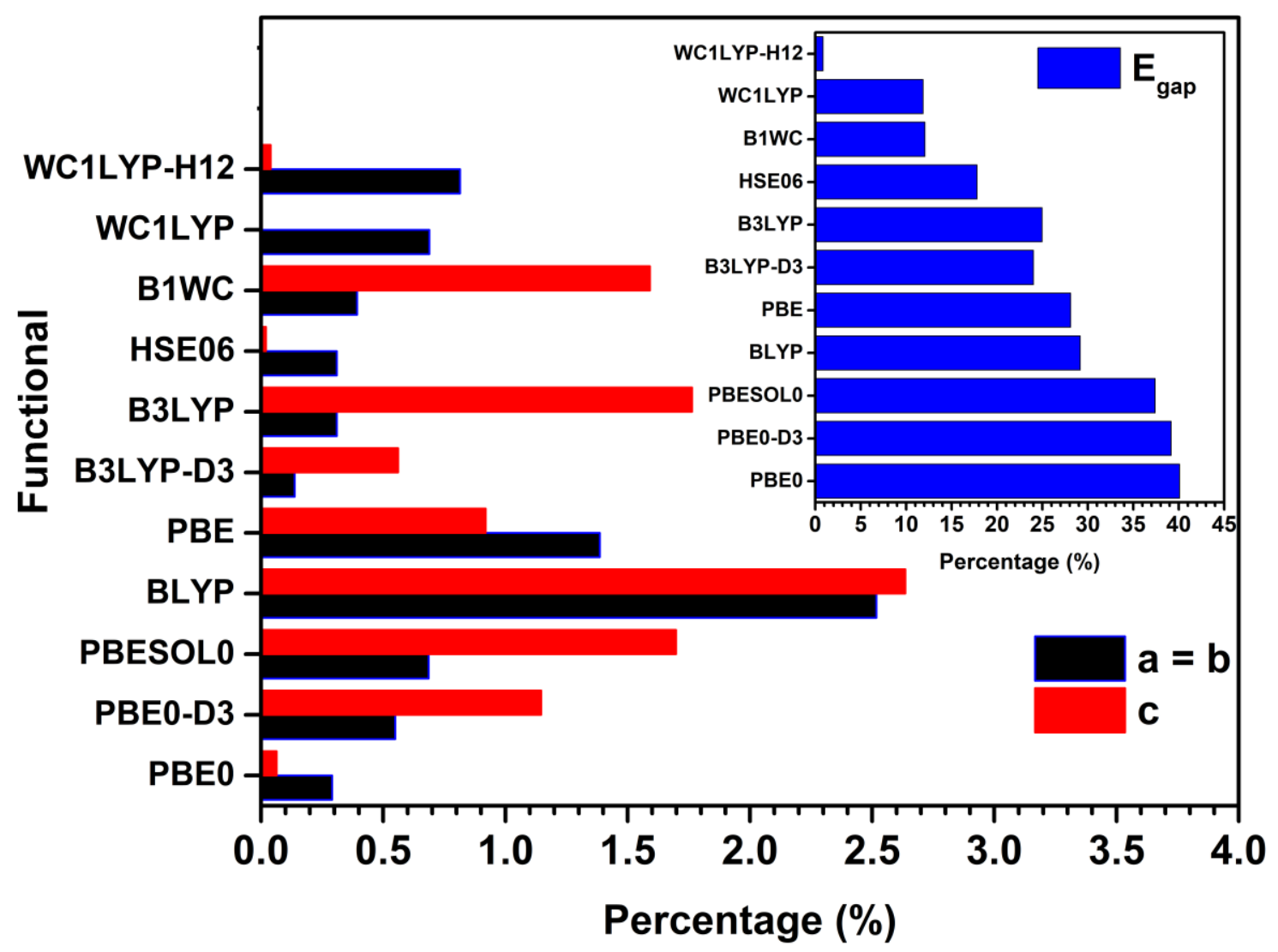

Figure S1: Calculated percentage difference for the lattice parameters and band gap energy for all probed functional. The experimental lattice parameters are defined as the reference. 
In Figure S2, it is described the convergence of the surface energy as the layer thickness increases for each studied surface.

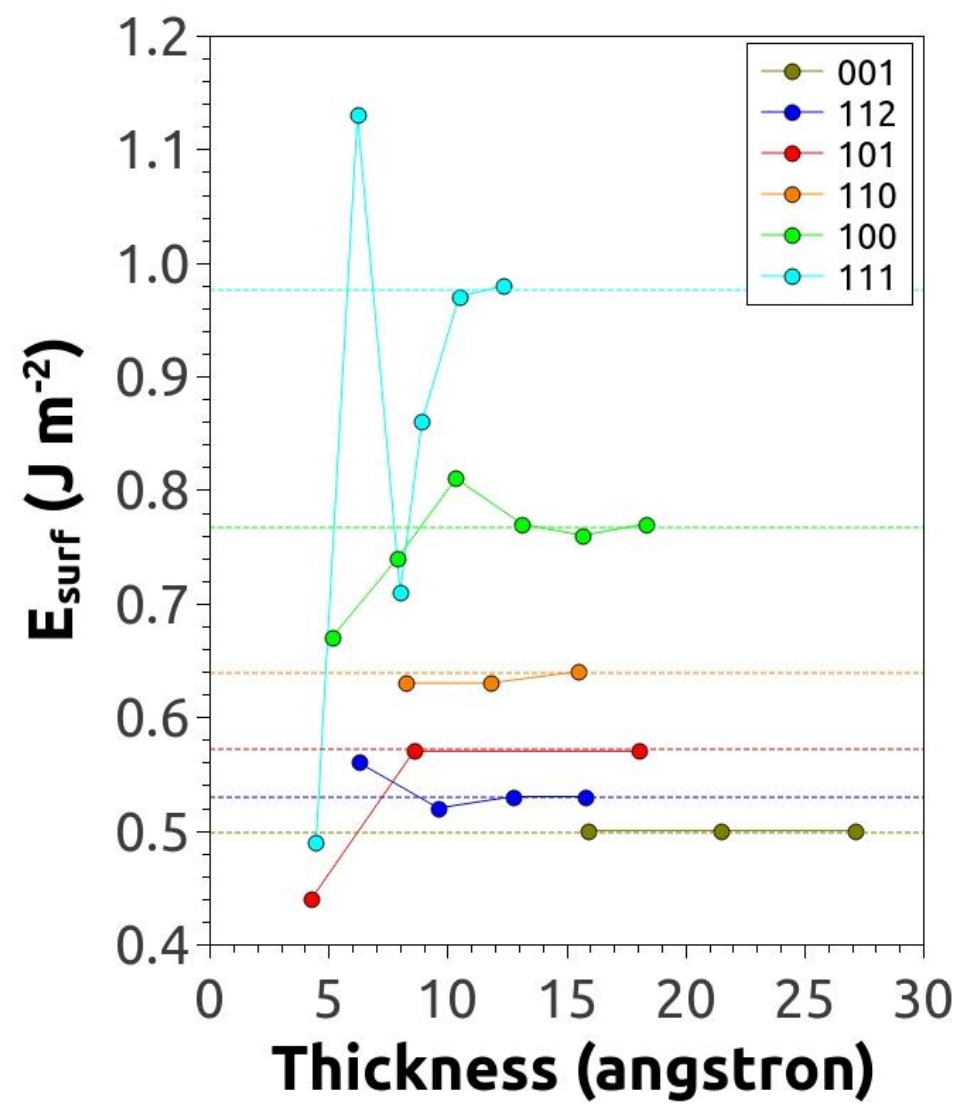

Figure S2 - The surface energy as a function of slab thickness of the (001), (112), (101), (110), (100) and (111) surfaces. 
Figure $\mathrm{S} 3$ shows the band structure and the density of states (DOS) of $\mathrm{CdMoO}_{4}$ bulk using WC1LYP(12\%).

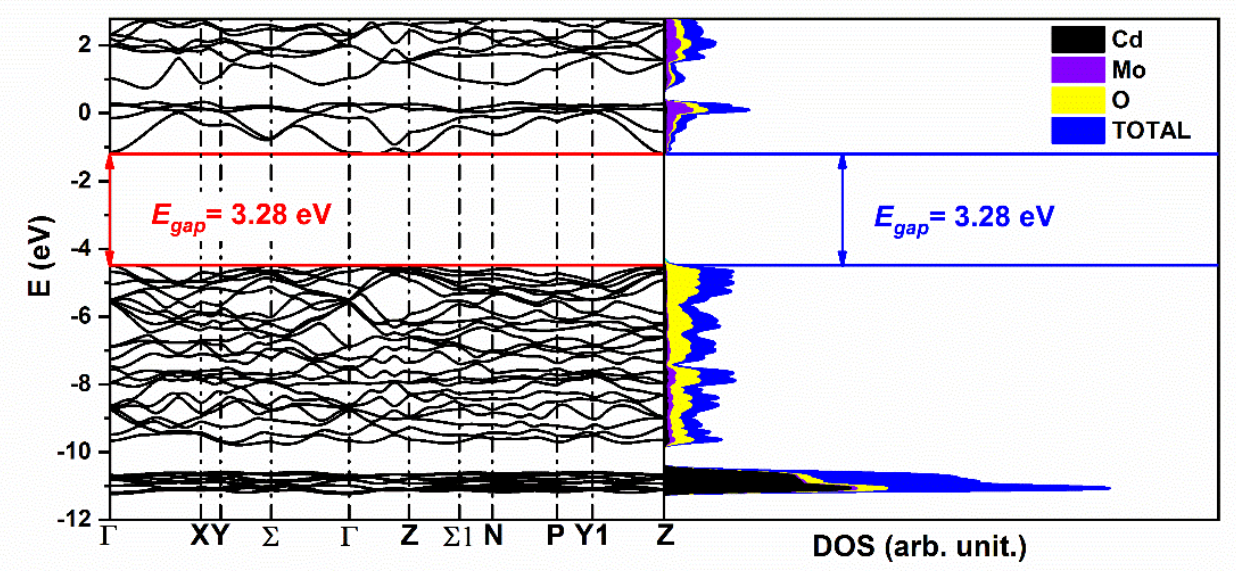

Figure S3: Band structure and density of states of the $\mathrm{CdMoO}_{4}$ bulk.

Figure S4 depicts the total energy evolution of a (1x1) and (2x2) (112) supercell surface through the optimization steps, and the respective migration of the oxygen to another position.
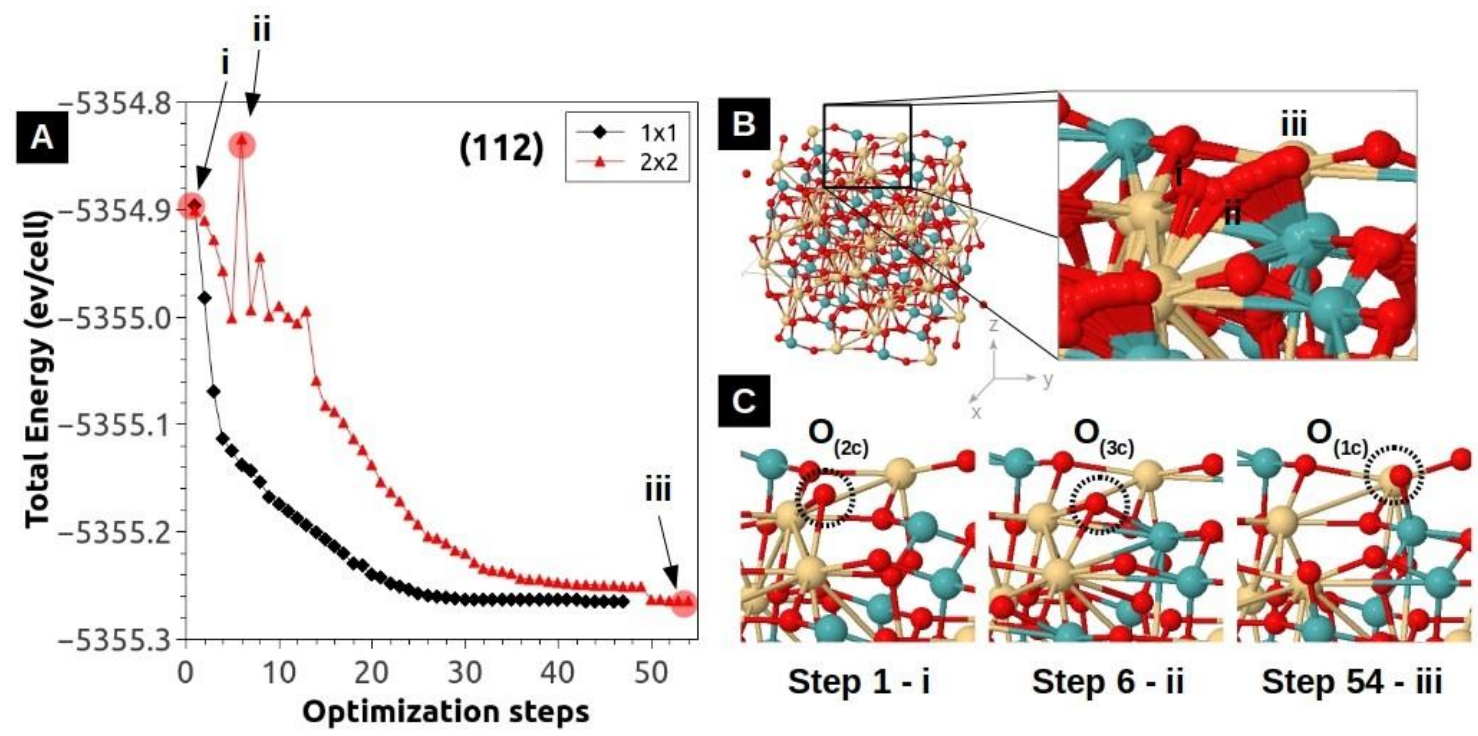

Figure S4 - (A) Total energy/cell optimization steps of (1x1) and (2x2) (112) surface. The total energy of the supercell was divided into 4, for a direct comparison with the (1x1) total energy. (B) The (2x2) supercell model, where the squared inset shows the snapshots of the oxygen (in red) migration from $\mathrm{Cd}$ (in yellow) to Mo (in green) polyhedron with the optimization. $(C)$ The initial geometry (i), the higher energy step (ii), and the final structure are marked (A) and (D), emphasizing the change in the oxygen coordination along the optimization. 


\section{Script for Nanoparticle density of states modeling and plotting}

The python script was done and used to plot the density of states of the nanoparticles from the calculated density of states of the surfaces.

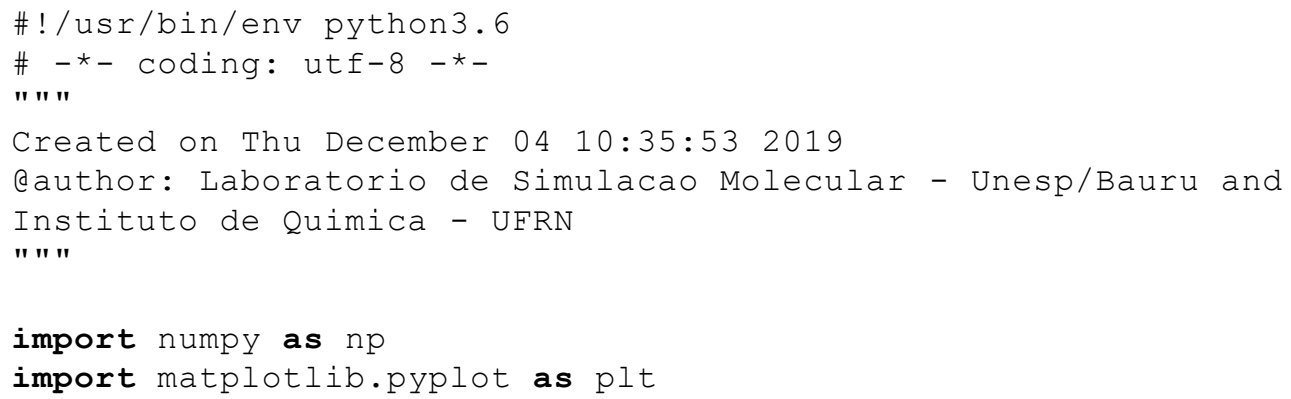




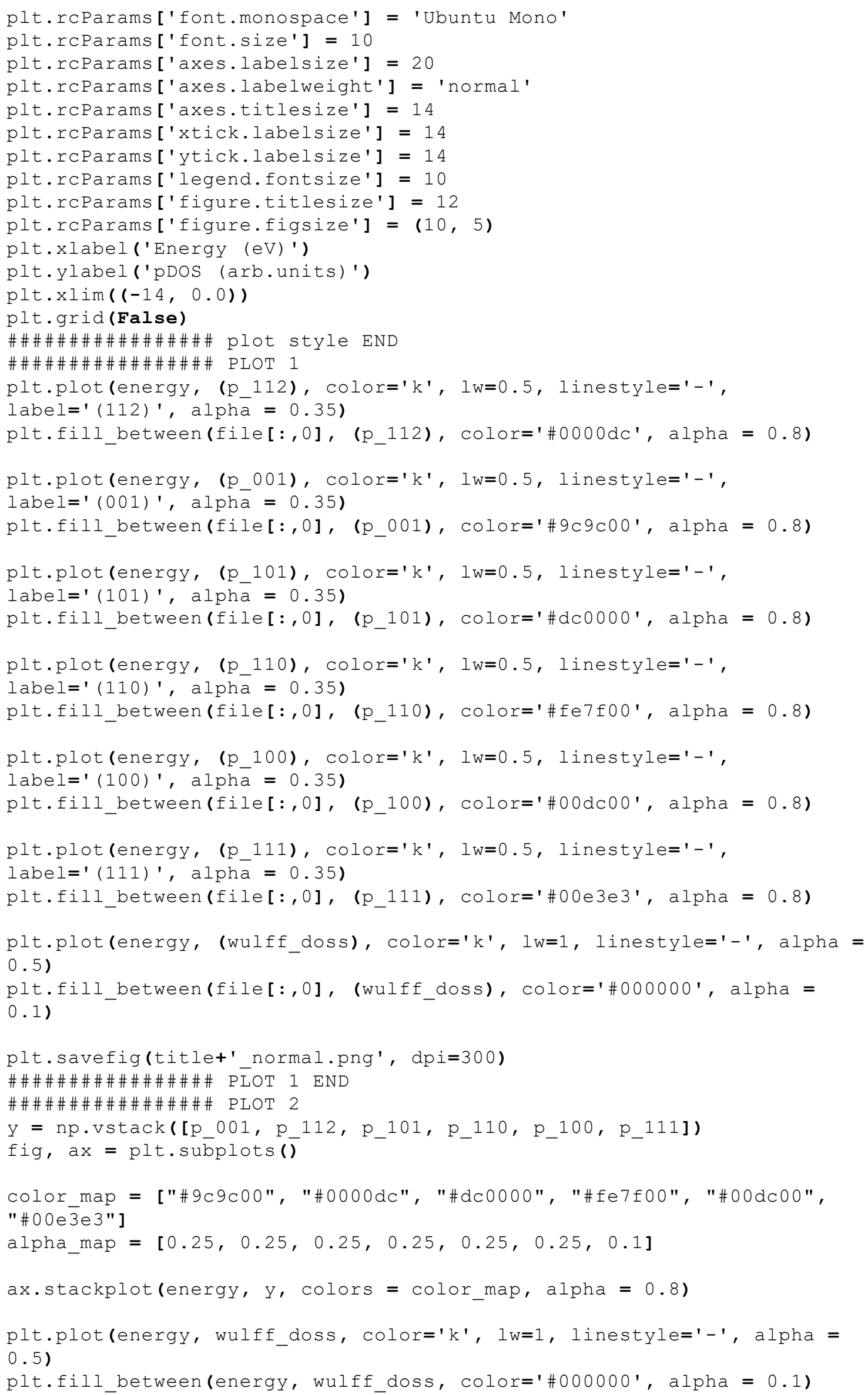


plt.xlabel ('Energy (eV)')

plt.ylabel('pDos (arb.units)')

plt.xlim( $(-14,0.0))$

plt.grid(False)

plt.savefig(title+'_stack.png', dpi=300)

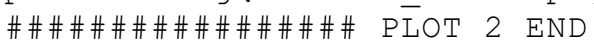

\section{Surface DOS used for Nanoparticle density of states modeling and plotting}

In the data below, the first column is the energy in $\mathrm{eV}$ and each one of the other columns represents the DOS of each surface.

$\begin{array}{rrrrrrr}-13.6877 & 0 & 0 & 0 & 0 & 0 & 0 \\ -13.6058 & 0 & 0 & 9863.5 & 0 & 1852.1 & 0 \\ -13.5236 & 747.16 & 0 & 10672 & 0 & 3010.1 & 0 \\ -13.4417 & 2972.3 & 1551.5 & 3968 & 0 & 4683.1 & 0 \\ -13.3595 & 2890.3 & 3034.3 & 0 & 1123.2 & 4236.6 & 0 \\ -13.2776 & 4059.4 & 2435.5 & 0 & 1609.4 & 3868.3 & 829.75 \\ -13.1954 & 4404.9 & 1789.9 & 0 & 1277.4 & 4826.7 & 366.65 \\ -13.1135 & 2593.8 & 1375 & 0 & 708.26 & 4892.9 & 2206.4 \\ -13.0314 & 2615.5 & 1553.3 & 0 & 1106.2 & 3845 & 2679.3 \\ -12.9495 & 2648.1 & 1520.5 & 0 & 1198.2 & 2968.5 & 2150.8 \\ -12.8673 & 3063.3 & 1874.2 & 0 & 535.44 & 6047.8 & 2417.4 \\ -12.7854 & 2892.4 & 1628.3 & 0 & 212.12 & 7829.7 & 2150.3 \\ -12.7032 & 2826.7 & 1153.6 & 0 & 529.51 & 6842.7 & 2734.8 \\ -12.6213 & 2695.2 & 1425.5 & 208.91 & 624.81 & 7979.1 & 3563.8 \\ -12.5391 & 3282.7 & 2459.6 & 1807.6 & 1595.4 & 7590.2 & 2745.6 \\ -12.4572 & 4428.1 & 3050.1 & 2070.5 & 1879.6 & 9011 & 2311 \\ -12.375 & 4983.1 & 3077.9 & 1831 & 2085.4 & 8721.8 & 3505.9 \\ -12.2931 & 5068 & 3310.1 & 2042.1 & 2726.2 & 6375 & 3765.8 \\ -12.2109 & 4941.4 & 2373.3 & 927.67 & 2611.3 & 6291.3 & 2852.3 \\ -12.129 & 5150.5 & 2653.5 & 1420.7 & 2262.4 & 6085.4 & 3385.6 \\ -12.0468 & 3633 & 2808.8 & 1434 & 2561.7 & 6949.1 & 4250.9 \\ -11.9649 & 3391.8 & 3206.9 & 956.97 & 2536.4 & 10694 & 4194.2 \\ -11.8828 & 4671.5 & 3879.8 & 1400.4 & 2771.7 & 12941 & 4297.2 \\ -11.8009 & 6403.2 & 4338.1 & 2080.3 & 2170.9 & 10737 & 4310.8 \\ -11.7187 & 8969.4 & 4365.3 & 1570.1 & 1889 & 9552.5 & 3925.1 \\ -11.6368 & 12750 & 5726.5 & 2017.3 & 2458.4 & 9106.8 & 3710.4 \\ -11.5546 & 11976 & 6006.5 & 2610.7 & 3033.6 & 5994.7 & 4062.6 \\ -11.4727 & 9300.3 & 5504.9 & 2609.5 & 2861.1 & 6029.7 & 5067.9 \\ -11.3905 & 7074.3 & 4845.2 & 2739.2 & 2227.1 & 6568.3 & 4681.4 \\ -11.3086 & 3356.3 & 3565.6 & 2662 & 2224 & 7195.8 & 4499.2 \\ -11.2264 & 1099.3 & 3013.4 & 3092.5 & 2293.3 & 8695.6 & 5942.9 \\ -11.1445 & 844.95 & 2603.4 & 2363.8 & 2939.5 & 7569.8 & 6004.2 \\ -11.0623 & 1766.9 & 944.33 & 2562.6 & 2188.1 & 6885.4 & 5060.4\end{array}$




\begin{tabular}{|c|c|c|c|c|c|c|}
\hline-10.9804 & 349.5 & 2676.3 & 3044.9 & 624.79 & 6723.3 & 2.8 \\
\hline-10.8983 & 3340.4 & 3350.7 & 3741.2 & 955.22 & 7694 & 4464.5 \\
\hline-10.8163 & 4246.1 & 2863 & 4242.8 & 1685.2 & 8943.9 & 5279.2 \\
\hline-10.7342 & 4617.2 & 3356.7 & 4784.1 & 2497.8 & 6717.9 & 3940.1 \\
\hline-10.6521 & 5839.4 & 4351 & 5093.1 & 2328.1 & 3951.9 & 5344.7 \\
\hline-10.5701 & 5775.7 & 3693.9 & 4439.8 & 1945 & 3725.3 & 6523.5 \\
\hline-10.4881 & 4377.5 & 3105 & 3377 & 1560.3 & 4566.9 & 5403.3 \\
\hline-10.406 & 4167.5 & 2381.7 & 1865.7 & 1710.2 & 6855.3 & 3617. \\
\hline-10.324 & 3533.2 & 1953.5 & 608.8 & 1914.2 & 7914.4 & 2527.6 \\
\hline-10.2419 & 3153.9 & 1908.5 & 423.57 & 2097.2 & 7918.8 & 4342.4 \\
\hline-10.1599 & 3922.2 & 1710.9 & 1890.8 & 1966.7 & 6947.6 & 4171.2 \\
\hline-10.0778 & 4108.2 & 2365.9 & 2968.1 & 2290.8 & 5667.3 & 2936.3 \\
\hline-9.9958 & 4090.5 & 5037.6 & 2554.8 & 1472.7 & 5824.6 & 3865.9 \\
\hline-9.9138 & 4372.1 & 5119.9 & 1669 & 1787.8 & 5704.9 & 3851.4 \\
\hline-9.8317 & 3809 & 4179.8 & 1708.9 & 2478.8 & 6459.4 & 3823.6 \\
\hline-9.7497 & 4342.9 & 4465.7 & 2210.7 & 3032 & 6925.4 & 3786.2 \\
\hline-9.6676 & 5619 & 4620.2 & 2442.2 & 2939.1 & 8883.7 & 3942.5 \\
\hline-9.5856 & 4484.6 & 3844.5 & 2652.2 & 2485.8 & 12098 & 5023.9 \\
\hline-9.5035 & 3242.4 & 3438.6 & 2006.8 & 2474.1 & 12317 & 4984 \\
\hline-9.4215 & 3630.4 & 3147.8 & 2631.7 & 2603 & 12465 & 4306.6 \\
\hline-9.3394 & 6977.7 & 3637.1 & 2336.6 & 2580 & 14285 & 4889 \\
\hline-9.2574 & 8195.1 & 3799.2 & 2089.2 & 2245.2 & 16733 & 4764.1 \\
\hline-9.1753 & 8785.6 & 4185.4 & 2550.7 & 2677.2 & 21353 & 4946.8 \\
\hline-9.0933 & 10572 & 3595 & 3357.3 & 2466.9 & 24081 & 6601.5 \\
\hline-9.0113 & 9111.2 & 4416.7 & 2618.8 & 2712.1 & 21776 & 7185.5 \\
\hline-8.9292 & 9923.1 & 4460 & 3143.6 & 3297.9 & 20167 & 6468.7 \\
\hline-8.8472 & 13506 & 3979.9 & 4190.2 & 3311 & 19342 & 5756.9 \\
\hline-8.7651 & 17373 & 4926.3 & 2887.9 & 3395.2 & 16899 & 5471.8 \\
\hline-8.6831 & 19289 & 6671.1 & 1935.4 & 4512.8 & 13510 & 7554.8 \\
\hline-8.601 & 16351 & 7265.9 & 1769.8 & 5329.5 & 8339.2 & 7228 \\
\hline-8.519 & 15011 & 6099.2 & 1587 & 4661.6 & 5452.9 & 6247.8 \\
\hline-8.4369 & 11824 & 6446.4 & 1589.6 & 4976.3 & 5515.4 & 7866.6 \\
\hline-8.3549 & 5957.4 & 5541.5 & 2648.5 & 3963.7 & 4200.7 & 8232.9 \\
\hline-8.2729 & 1597.5 & 2384.3 & 3088 & 2446.2 & 1982.1 & 6403.2 \\
\hline-8.1908 & 0 & 1482.3 & 3283 & 1119.6 & 357.74 & 5394.7 \\
\hline-8.1088 & 0 & 995 & 4099.4 & 1342.9 & 0 & 6239.1 \\
\hline-8.0267 & 0 & 0 & 4666 & 559.77 & 0 & 7204.8 \\
\hline-7.9447 & 0 & 0 & 5600 & 0 & 0 & 4305.3 \\
\hline-7.8626 & 0 & 0 & 5992.5 & 0 & 0 & 1771.3 \\
\hline-7.7806 & 0 & 0 & 6897.5 & 0 & 0 & 1831.4 \\
\hline-7.6985 & 0 & 0 & 7213.2 & 0 & 0 & 391.43 \\
\hline-7.6165 & 0 & 0 & 7817.5 & 0 & 0 & 1733.1 \\
\hline-7.5345 & 0 & 0 & 6919.6 & 0 & 0 & 1480.2 \\
\hline-7.4524 & 0 & 0 & 4021.2 & 0 & 0 & 995 \\
\hline-7.3703 & 0 & 0 & 1069.7 & 0 & 0 & 0 \\
\hline-7.2883 & 0 & 0 & 0 & 0 & 0 & \\
\hline
\end{tabular}




\begin{tabular}{|c|c|c|c|c|c|c|}
\hline-7.2063 & 0 & 0 & 0 & 0 & 0 & 0 \\
\hline-7.1242 & 0 & 0 & 0 & 0 & 0 & 0 \\
\hline-7.0422 & 0 & 0 & 0 & 0 & 0 & 0 \\
\hline-6.9601 & 0 & 0 & 0 & 0 & 0 & 0 \\
\hline-6.8781 & 0 & 0 & 0 & 0 & 0 & 6 \\
\hline-6.796 & 0 & 0 & 0 & 0 & 0 & 0 \\
\hline-6.714 & 0 & 0 & 0 & 0 & 0 & 0 \\
\hline-6.632 & 0 & 0 & 0 & 0 & 0 & \\
\hline-6.5499 & 0 & 0 & 0 & 0 & 0 & 0 \\
\hline-6.4679 & 0 & 0 & 0 & 0 & 0 & 0 \\
\hline-6.3858 & 0 & 0 & 0 & 0 & 0 & 0 \\
\hline-6.3038 & 0 & 0 & 0 & 0 & 0 & 0 \\
\hline-6.2217 & 0 & 0 & 0 & 0 & 0 & \\
\hline-6.1397 & 0 & 0 & 0 & 0 & 0 & \\
\hline-6.0576 & 0 & 0 & 0 & 0 & 0 & \\
\hline-5.9756 & 0 & 0 & 0 & 0 & 0 & 0 \\
\hline-5.8935 & 0 & 0 & 0 & 0 & 0 & \\
\hline-5.8115 & 0 & 0 & 0 & 0 & 0 & \\
\hline-5.7295 & 0 & 0 & 0 & 0 & 0 & \\
\hline-5.6474 & 0 & 0 & 0 & 0 & 0 & \\
\hline-5.5654 & 0 & 0 & 0 & 0 & 0 & \\
\hline-5.4834 & 0 & 0 & 0 & 0 & 0 & \\
\hline-5.4012 & 0 & 0 & 0 & 0 & 0 & \\
\hline-5.3193 & 0 & 0 & 0 & 0 & 0 & \\
\hline-5.2371 & 0 & 0 & 0 & 0 & 0 & 0 \\
\hline-5.1552 & 0 & 0 & 0 & 0 & 0 & \\
\hline-5.0731 & 0 & 0 & 0 & 0 & 107.27 & 0 \\
\hline-4.9912 & -3.22 & 0 & 0 & 0 & 282.97 & \\
\hline-4.909 & 94.03 & 0 & 0 & 0 & 13.2 & 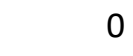 \\
\hline-4.8271 & 136.08 & 0 & 0 & 0 & 595.1 & 0 \\
\hline-4.7449 & 95.67 & 264.85 & 0 & 0 & 84.13 & 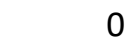 \\
\hline-4.663 & 167.61 & 4.01 & 0 & 94.28 & 426.25 & 0 \\
\hline-4.5808 & 222.13 & 183.54 & 0 & -28.03 & 553.4 & \\
\hline-4.4989 & 263.55 & -21.13 & 0 & 180.47 & 1015 & 0 \\
\hline-4.4167 & 213.01 & 348.58 & 0 & 7.05 & 2709.6 & 284.75 \\
\hline-4.3348 & 380.28 & 311.99 & 0 & 136.24 & 3182.2 & 312.96 \\
\hline-4.2526 & 441.82 & 232.67 & 0 & 286.1 & 5852.5 & 250.7 \\
\hline-4.1707 & 546.53 & 318.23 & 0 & 435.9 & 6888.5 & 83.37 \\
\hline-4.0885 & 557.41 & 348.33 & 0 & 217.94 & 5542.8 & 1759 \\
\hline-4.0066 & 582.64 & 869.18 & 0 & 204.77 & 5114.2 & 1468 \\
\hline-3.9245 & 1774 & 760.23 & 0 & 251.74 & 7676.8 & 1551.2 \\
\hline-3.8426 & 2737.6 & 1577.5 & 129.78 & 857.49 & 7510.6 & 2115.5 \\
\hline-3.7604 & 5405.7 & 2524.5 & 76.22 & 1286.6 & 2836.6 & 2199.5 \\
\hline-3.6785 & 9063 & 4083.8 & 79.91 & 1878.7 & 666.26 & 2456.7 \\
\hline-3.5963 & 7642.5 & 5339.8 & 281.65 & 3953.9 & -145.16 & 3087.2 \\
\hline-3.5141 & 3823.2 & 3741.6 & 85.69 & 3252.7 & 892.49 & 4470.3 \\
\hline
\end{tabular}




\begin{tabular}{|c|c|c|c|c|c|c|}
\hline 22 & 1357 & 1451.7 & 182.28 & .96 & 1781.7 & 706.7 \\
\hline-3.35 & 47.68 & 280.9 & 450.41 & 0 & 3544.8 & 1887.8 \\
\hline .2681 & 75.73 & 22.79 & 231.83 & 1136.7 & 3108.4 & 03.2 \\
\hline .1859 & 29.49 & 113.3 & 657.14 & 582.62 & 2864.8 & 2770.3 \\
\hline-3.104 & 34.64 & 400.3 & 259.12 & 339.48 & 1560.6 & 028.2 \\
\hline 3.0218 & 23.35 & 11.88 & 43.79 & 764.59 & 1616.3 & 425.86 \\
\hline 2.9399 & 323.41 & 49.45 & 460 & 484.6 & 1822.5 & 739.64 \\
\hline 2.8578 & 74.65 & 397.91 & 1763.1 & 0 & 983.6 & 405.39 \\
\hline 2.7759 & 27.72 & 73.69 & 3189.5 & 1318 & 633.79 & 3173 \\
\hline 2.6937 & 99.68 & 49.14 & 4371.9 & 818.32 & 1168.3 & 453.9 \\
\hline 2.6118 & 57.25 & 87.74 & 3198.4 & 47 & 396.93 & 793.68 \\
\hline-2.5296 & 465.63 & 330.47 & 918.68 & 320.58 & 777.72 & 359.97 \\
\hline-2.4477 & 332.27 & 276.09 & 1860.2 & 432.31 & 2691 & 615.71 \\
\hline 2.3655 & 04.34 & 243.13 & 547.23 & 192.28 & 3523.2 & 2109.6 \\
\hline 2.2836 & 96.65 & 59.47 & 0 & 164.39 & 3202.2 & 1824 \\
\hline 2014 & 651.6 & 267.59 & 0 & 185.5 & 5072.2 & 2532.5 \\
\hline 2.1195 & 2992.5 & 2170.6 & 22 & 271.84 & 6935.2 & 3172.3 \\
\hline 2.0373 & 20 & 20 & & & 3.7 & 99.6 \\
\hline-1.9554 & 728.9 & 1612.9 & 528.17 & 350.04 & 5056.4 & 1828.9 \\
\hline 1.87 & 66.6 & 37.8 & 756 & .4 & .8 & 191.4 \\
\hline 1.7913 & 651.4 & 3046.3 & 291.2 & $22 \epsilon$ & 5796.9 & 1470.5 \\
\hline 1.7092 & 4045.1 & 3473.6 & 321.39 & 3058.4 & 863.3 & 750.67 \\
\hline-1.6273 & 36.1 & 2461.2 & 30.5 & 255 & 4622.5 & 1997.2 \\
\hline-1.5451 & 660.3 & 2551.5 & 510.62 & 1700.4 & 3914.1 & 1657.2 \\
\hline 1.4632 & 32.6 & 3259.2 & 337.35 & 1664.1 & 6649.6 & 2032.4 \\
\hline-1.381 & 795.8 & 2612.2 & 296.52 & 1625.2 & 6634 & 1574.4 \\
\hline 1.2991 & 700.9 & 2327.5 & 96.58 & 1782 & 3324.9 & 1997.4 \\
\hline-1.2169 & 041.9 & 2549.2 & 563.29 & 1903.2 & 2979.6 & 3357.1 \\
\hline-1.135 & 85.22 & 1612.7 & 1594.8 & 1717.1 & 471.8 & 3496 \\
\hline 1.0528 & . & 09.4 & 1707.8 & & 00.6 & 2711.5 \\
\hline-0.9709 & 71.62 & 12.51 & 1489.3 & 340.63 & 223 & 1879.3 \\
\hline-0.8887 & 438.68 & & 2496.3 & & & 1644.3 \\
\hline 0.8068 & 492.64 & 557.78 & 2728.6 & 224.51 & 248.42 & 1070.3 \\
\hline 0.7246 & 14.05 & 65.34 & 1851.5 & 278.12 & 2147.4 & 1434.1 \\
\hline .6427 & 79.65 & 1759.3 & & & & 1604.6 \\
\hline-0.5606 & 608.43 & 1464.8 & 1595.2 & 984.77 & 3582.9 & 2495.4 \\
\hline-0.4787 & 93.7 & 6.36 & 2158.6 & 746.66 & 5087 & 1595.2 \\
\hline-0.3965 & 61.4 & 6.98 & .6 & 38 & 3.3 & 1509.6 \\
\hline-0.3140 & 2005 & & 1892.1 & 342.68 & 5.6 & 872.94 \\
\hline-0.2324 & 359.5 & 1803.8 & 1849.1 & 597.59 & 3833.8 & 482.99 \\
\hline-0.1505 & 1225.7 & 801.33 & 1229.3 & 452.33 & 1654.2 & 949.35 \\
\hline-0.0683 & 791.71 & 320.27 & 662.84 & 1028.6 & 1016.9 & 1812.1 \\
\hline 0.0136 & 519.16 & 652.54 & 356.54 & 1119.2 & 569.4 & 1426.8 \\
\hline 0.0958 & 273.05 & 61.83 & 88.86 & 760.7 & 104.86 & 1203.7 \\
\hline 0.1777 & 249.72 & 173.04 & 287.2 & 235.8 & 223.65 & 1174.9 \\
\hline 599 & 190.45 & 128.84 & 49.69 & .54 & 0 & 925.02 \\
\hline
\end{tabular}




$\begin{array}{rrrrrrr}0.3418 & 122.76 & 63.24 & 223.31 & 96.73 & 0 & 762.78 \\ 0.424 & 85.94 & 109.48 & 309.88 & -3.15 & 0 & 587.01 \\ 0.5059 & 69.83 & 0 & 450.24 & 0 & 0 & 0 \\ 0.588 & -5.01 & 0 & 512.68 & 0 & 0 & 0 \\ 0.6699 & 0 & 0 & 569.41 & 0 & 0 & 0 \\ 0.7521 & 0 & 0 & 486.05 & 0 & 0 & 109.21 \\ 0.834 & 0 & 0 & 245.91 & 0 & 0 & 0 \\ 0.9162 & 0 & 0 & 531.46 & 0 & 2.38 & 711.78 \\ 0.9981 & 14.38 & 0 & 85.61 & 0 & 576.36 & 0 \\ 1.0803 & 30.31 & 0 & 295.09 & 0 & 1525.8 & 566.56 \\ 1.1622 & 66.67 & 12.6 & -0.25 & 0 & 724.2 & 1188.2 \\ 1.2444 & 65.9 & 67.23 & 58.53 & 0 & 871.39 & 0 \\ 1.3263 & 63.09 & 298.94 & 100.04 & 226.23 & 944.03 & 0 \\ 1.4085 & 116 & 362 & 19.84 & 161.32 & 747.48 & 0 \\ 1.4904 & 188.26 & 525.18 & 0 & 349.01 & 99.6 & 0 \\ 1.5726 & 313.4 & 238.78 & 0 & 359.19 & 276.38 & 99.02 \\ 1.6545 & 379.19 & 94.94 & 0 & 306.7 & 994.2 & 325.42 \\ 1.7366 & 303.49 & 214.05 & 0 & 147.64 & 552.35 & 494.06 \\ 1.8188 & 275.92 & 254.32 & 0 & 257.66 & 147.71 & 589.85 \\ 1.9007 & 298.29 & 238.35 & 0 & 190.44 & 500.62 & 999.79 \\ 1.9829 & 336.85 & 115.21 & 0 & 205.44 & 110.96 & 765.34 \\ 2.0648 & 265.59 & 131.12 & 157 & 94.37 & 110.38 & 256.94 \\ 2.147 & 145.69 & 55.85 & 538.89 & 459.13 & 991.34 & 351.09 \\ 2.2289 & 87.04 & 148.82 & 255.42 & 222.74 & 977.67 & 423.61 \\ 2.3111 & 113.6 & 261.6 & 199.82 & 179.87 & 294.45 & 410.83 \\ 2.393 & 157.58 & 312.11 & -45.17 & -4.49 & 1500.9 & 772.04 \\ 2.4752 & 252.58 & 440.53 & 96.1 & 143.79 & 836.35 & 314.67 \\ 2.5571 & 163.35 & 355.51 & 284.94 & 267.6 & 1166.1 & 525.55 \\ 2.6393 & 338.61 & 625.03 & 65.24 & 233.81 & 1149 & 293.94 \\ 2.7212 & 270.58 & 299.7 & 494.16 & 309.39 & 489.99 & 519.4 \\ 2.8033 & 0 & 0 & 0 & 0 & 0 & 0\end{array}$

